\title{
DYNAMICS OF A QUANTUM PARTICLE IN ASYMMETRIC BISTABLE POTENTIAL WITH ENVIRONMENTAL NOISE
}

\author{
P. CALDARA*, A. LA COGNATA, D. VALENTI ${ }^{\dagger}$ and B. SPAGNOLO \\ Dipartimento di Fisica e Tecnologie Relative, Università di Palermo, \\ Group of Interdisciplinary Physics and CNISM-INFM, Unita di Palermo, \\ Viale delle Scienze, Ed.18, I-90128 Palermo, Italy \\ *pcaldara@gip.dft.unipa.it \\ ${ }^{\dagger}$ valentid@gip.dft.unipa.it
}

M. BERRITTA, E. PALADINO and G. FALCI

Dipartimento di Metodologie Fisiche e Chimiche (DMFCI), Università di Catania, Viale A. Doria 6, I-95125 Catania, Italy CNR-IMM MATIS, Catania, Italy

Received 24 August 2010

\begin{abstract}
In this work we analyze the dynamics of a quantum particle subject to an asymmetric bistable potential and interacting with a thermal reservoir. We obtain the time evolution of the population distributions in both energy and position eigenstates of the particle, for different values of the coupling strength with the thermal bath. The calculation is carried out using the FeynmanVernon functional under the discrete variable representation.
\end{abstract}

Keywords: Bistable potential; NES; DVR; Caldeira-Leggett model.

\section{Introduction}

A feature which makes a strong difference-between the behaviour of a quantum system with respect to a classical one is quantum tunneling. This effect often occurs in condensed matter physics, such as Josephson junctions and hetero-nanostructures. ${ }^{1,2}$ In a dissipative quantum system interacting with a thermal bath, the quantum tunneling can play an important role on the relaxation time from a metastable state. ${ }^{3}$ During the last few years, the effects of environment on quantum tunneling phenomenon have been intensively studied. ${ }^{4-7}$ Commonly, environment is modelled as a number $\mathcal{N}$ (usually $\mathcal{N} \rightarrow \infty$ ) of harmonic oscillators considered at thermal equilibrium, i.e. thermal bath, interacting with the quantum system through a bilinear coupling. ${ }^{8-12}$ In this context, symmetric and asymmetric quantum bistable systems are good enough to analyze superconducting quantum bits and decoherence 
phenomena. $^{13,14}$ Obtaining longer coherence times in such systems, when they interact with noisy environment, is one of the major requirements in devising and manufacturing devices capable of storing quantum bits. In this respect, a main topic is to know the properties of a particle subject to an external potential, in the presence of random fluctuations. It can also be useful to study the changes occurring in the dynamics of a quantum particle affected by noisy perturbations, when different shapes of the potential profile are used. Potentials which model the interaction with laser beams have most interesting implications for quantum systems such as the coherent destruction of tunneling, ${ }^{15}$ the effect of quantum stochastic resonance, ${ }^{16}$ and the control and reduction of decoherence in open quantum systems. ${ }^{17}$ In this work, in order to analyze the evolution of a quantum particle subject to time-independent asymmetric bistable potential and affected by environmental noise, we use the Caldeira-Leggett model, ${ }^{4}$ which allows us to derive a quantum mechanical analogue of the generalized Langevin equation. The study is performed by using the approach of the Feynman-Vernon functional ${ }^{18}$ in discrete variable representation (DVR). ${ }^{19,20}$

\section{The Model}

Our system consists of a quantum particle with mass $M$, interacting with a thermal bath which plays the role of environment. The dynamics of the particle is investigated by using the Caldeira-Leggett model. ${ }^{4}$ In our analysis, $\hat{q}$ and $\hat{p}$ are one-dimensional operators for position and momentum, respectively.

The unperturbed Hamiltonian of the system is

$$
\hat{H}_{0}=\frac{\hat{p}^{2}}{2 M}+\hat{V}_{0}(\hat{q})
$$

where

$$
\hat{V}_{0}(\hat{q})=\frac{M^{2} \omega_{0}^{4}}{64 \Delta U} \hat{q}^{4}-\frac{M \omega_{0}^{4}}{4} \hat{q}^{2}-\hat{q} \epsilon
$$

is the asymmetric bistable potential shown in Fig. 1. Here, $\epsilon$ and $\Delta U$ are the asymmetry parameter and the barrier height, respectively, and $\omega_{0}$ is the natural oscillation frequency. In our study we consider only 8 energy eigenstates. In Fig. 1 these energy eigenvalues are shown on the vertical axis. In the same figure, on the horizontal axis, we indicate the 8 position eigenvalues, obtained by using the DVR-state $\left|q_{\mu}\right\rangle$. The black circle marks the initial position of the particle, that is the system at $t=0$ is in a state given by a proper linear combination of the 8 eigenstates $\left|q_{\mu}\right\rangle$ considered in our analysis. The curves shown in the figures are the eigenfunctions corresponding to the 8 energy eigenvalues.

In order to describe the dynamics of the particle interacting with environment, we consider the following Hamiltonian

$$
\hat{H}(t)=\hat{H}_{0}(t)+\hat{H}_{B},
$$




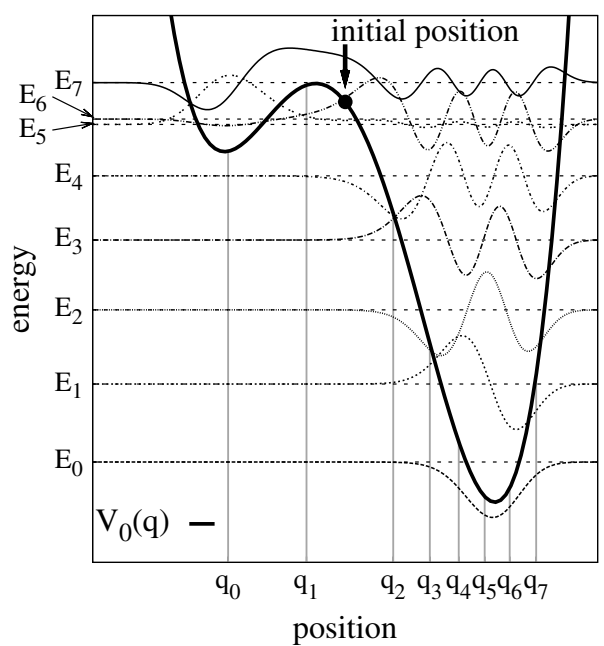

Fig. 1. Potential profile $V_{0}(q)$ (see Eq. (2)) for $\Delta U=3$ and $\epsilon=0.5$. Energy levels and corresponding eigenstates considered in our analysis are indicated by horizontal lines and curves, respectively. The energy eigenvalues are $E_{0}=-2.01, E_{1}=-0.92, E_{2}=0.11, E_{3}=1.08, E_{4}=1.97, \quad E_{5}=2.69, \quad E_{6}=2.76$, $E_{7}=3.27$. By using the DVR-state $\left|q_{\mu}\right\rangle$, eigenvalues of the position operator are obtained and shown on the horizontal axis: $q_{0}=-4.17, q_{1}=-1.38, q_{2}=1.71, q_{3}=3.02, q_{4}=4.05, q_{5}=4.97, q_{6}=5.86, q_{7}=6.81$. The initial position is $q_{\text {start }}=0$ (black circle).

where

$$
\hat{H}_{B}=\sum_{j=1}^{\mathcal{N}} \frac{1}{2}\left[\frac{\hat{p}_{j}^{2}}{m_{j}}+m_{j} \omega_{j}^{2}\left(\hat{x}_{j}-\frac{c_{j}}{m_{j} \omega_{j}^{2}} \hat{q}\right)^{2}\right]
$$

is the Hamiltonian which describes the thermal reservoir and its interaction with the particle. As usual, the thermal bath is depicted by an ensemble of $\mathcal{N}$ harmonic oscillators with spatial coordinate $\hat{x}_{j}$, momentum $\hat{p}_{j}$, mass $m_{j}$, and frequency $\omega_{j}$. The coefficients $c_{j}$ are the coupling constant between system and thermal bath.

We note that, as $\mathcal{N} \rightarrow \infty$, from Eq. (4) a continuous spectral density is obtained.

In our study we use the Ohmic spectral density characterized by an exponential cut-off $\omega_{c}$

$$
J(\omega)=\eta \omega \exp \left(-\frac{\omega}{\omega_{c}}\right)
$$

Here, $\eta=M \gamma$ with $\gamma$ the strength of the coupling between system and heat bath. We note also that $\omega_{c} \gg \omega_{0}, \omega_{j}, \gamma$.

Because of the bilinear coupling between the coordinate $\hat{q}$ of the system and the coordinate $\hat{x}$ of the thermal bath, this model is the quantum analogue of a classical system affected by a constant random force. ${ }^{7}$ In the next two subsections we briefly summarize the mathematical approach used in this study. 


\subsection{The Feynman-Vernon approach}

In order to make our analysis independent on the properties of the heat bath, we trace out the degrees of freedom of the reservoir by using the reduced density operator

$$
\rho\left(q_{f}, q_{f}^{\prime} ; t\right)=\int d q_{0} \int d q_{0}^{\prime} K\left(q_{f}, q_{f}^{\prime}, t ; q_{0}, q_{0}^{\prime}, t_{0}\right) \rho_{S}\left(q_{0}, q_{0}^{\prime}, t_{0}\right),
$$

where the propagator $K$ is given by

$$
K\left(q_{f}, q_{f}^{\prime}, t ; q_{0}, q_{0}^{\prime}, t_{0}\right)=\int_{q\left(t_{0}\right)=q_{0}}^{q(t)=q_{f}} \mathcal{D} q \int_{q^{\prime}\left(t_{0}\right)=q_{0}^{\prime}}^{q^{\prime}(t)=q_{f}^{\prime}} \mathcal{D} q^{\prime} \mathcal{A}[q] \mathcal{A}^{*}\left[q^{\prime}\right] \mathcal{F}_{F V}\left[q, q^{\prime}\right]
$$

and $\mathcal{A}[q]=\exp \left(i\left(S_{S}[q] / \hbar\right)\right)$ with $S_{S}[q]$ being the classical action functional. In Eq. (7), $\mathcal{F}_{F V}\left[q, q^{\prime}\right]=\exp \left(-\left(\phi_{F V}\left[q, q^{\prime}\right] / \hbar\right)\right)$ is the Feynman-Vernon $(\mathrm{FV})$ influence functional with the influence weight functional $\phi_{F V}\left[q, q^{\prime}\right]$ depending on the bath correlation function. ${ }^{7}$

\subsection{Discrete variable representation}

By solving the eigenvalue equation connected with the Hamiltonian $\hat{H}_{0}$ (see Eq. (1)), we get the energy eigenstates (see vertical axis in Fig. 2). Within the framework of the discrete variable representation $(\mathrm{DVR})^{19}$ it is possible to obtain the basis $\left\{\left|q_{\mu}\right\rangle\right\}$ of eigenstates of the position operator $\hat{q}$ (see horizontal axis in Fig. 2).
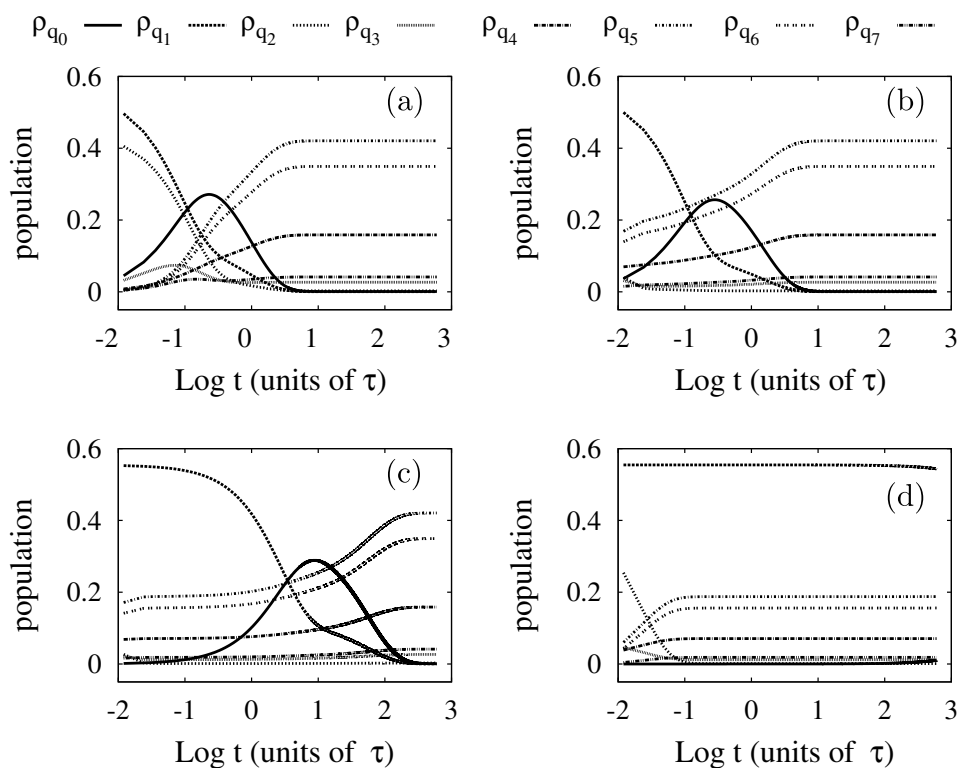

Fig. 2. Time evolution of the diagonal elements, $\rho_{q_{\mu}}(\mu=0,1, \ldots, 7)$, of the density matrix in q-representation. The matrix elements $\rho_{q_{\mu}}$ are the population distributions in the eight position eigenstates considered. The time evolution is obtained for different values of the coupling strength: (a) $\eta=0.01$, (b) $\eta=0.4$, (c) $\eta=1$ and (d) $\eta=2.8$. 
In this representation, using Eq. (7), the continuous real-time path integral given in Eq. (6) becomes a discrete path with $m$ transitions at times $t_{1}, t_{2}, \ldots, t_{m}$

$$
\rho_{\mu_{m} \nu_{m}}(t)=\sum_{\mu_{0} \nu_{0}} \int_{\xi\left(t_{0}\right)=\xi_{0}}^{\xi(t)=\xi_{m}} \mathcal{D} \xi \int_{\chi\left(t_{0}\right)=\chi_{0}}^{\chi(t)=\chi_{m}} \mathcal{D} \chi \mathcal{C}[\xi, \chi] \mathcal{F}_{F V}[\xi, \chi] \rho_{\mu_{0} \nu_{0}}
$$

where $\mathcal{C}[\xi, \chi]=\mathcal{A}[q] \mathcal{A}^{*}\left[q^{\prime}\right]$ and the influence weight functional of the FV functional is

$$
\phi_{F V}[\xi, \chi]=-\sum_{l=1}^{m} \sum_{j=0}^{l-1} \xi_{l} S\left(t_{l}-t_{j}\right) \xi_{j}-i \sum_{l=1}^{m} \sum_{j=0}^{l-1} \xi_{l} R\left(t_{l}-t_{j}\right) \chi_{j} .
$$

Here, the absolute coordinates $q_{j}$ are replaced by the discrete relative coordinates $\xi_{j}(t)=q_{j}(t)-q_{j}^{\prime}(t)$ and center of mass coordinates $\chi_{j}=q_{j}(t)+q_{j}^{\prime}(t)$.

Because we are interested in the evolution of the populations, in Eq. (8) we consider the diagonal terms $\rho_{\mu_{m} \mu_{m}}(t)$. Applying the non-interacting cluster approximation (NICA), ${ }^{20}$ we get the following master equation (ME)

$$
\rho_{\mu \mu}(t)=\sum_{\nu=1}^{N} \int_{t_{0}}^{t} d t^{\prime} \mathcal{H}_{\mu \nu}\left(t-t^{\prime}\right) \rho_{\nu \nu}\left(t^{\prime}\right), \quad \mu=1, \ldots, N,
$$

where $N$ is the number of eigenstates and the kernel $\mathcal{H}$, which indicates the cluster matrix, takes into account of all possible transitions in the DVR paths. ${ }^{3}$

According to the path integral technique based on the Feynman-Vernon theory, using ME corresponds to take into account only the paths connecting diagonal elements of the reduced density matrix of the position operator $\hat{q} \cdot{ }^{20}$

Within NICA we neglect all intercluster interactions. We assume furthermore that the characteristic memory time $\tau_{\text {mem }}$ of the matrix elements of $\mathcal{H}$ in Eq. (10) is the smallest time scale of the problem (Markovian limit). By this assumption we obtain the following Markovian approximated master equation

$$
\dot{\rho}_{\mu \mu}(t)=\sum_{\nu=1}^{N} \Gamma_{\mu \nu}(t) \rho_{\nu \nu}(t)
$$

with the time-dependent rate coefficients

$$
\Gamma_{\mu \nu}(t)=\int_{0}^{\infty} d \tau \mathcal{H}_{\mu \nu}(t, t-\tau)
$$

Since the diagonal elements $\rho_{\mu \mu}(t)$ obey Eq. (11), the long-time dynamics is ruled by a single exponential decay. Thus, Eq. (11) is a set of coupled ordinary first-order differential equations, which can be decoupled via a diagonalization procedure. The diagonalized rate matrix reads

$$
\sum_{\kappa_{1}, \kappa_{2}=1}^{N}\left(S^{-1}\right)_{\mu \kappa_{1}} \Gamma_{\kappa_{1} \kappa_{2}} S_{\kappa_{2} \nu}=\Lambda_{\mu} \delta_{\mu \nu}
$$


where $S_{\mu \nu}$ denotes the element of the transformation matrix and $\Lambda_{\mu}$ the eigenvalues of the rate matrix. The general solution of the Markov approximated ME is

$$
\rho_{\mu \mu}(t)=\sum_{\nu, \kappa=1}^{N} S_{\mu \nu}\left(S^{-1}\right)_{\mu \kappa} e^{\Lambda_{\nu}\left(t-t_{0}\right)} \rho_{\kappa \kappa}\left(t_{0}\right) .
$$

Because of the conservation probability, for the diagonal matrix elements holds

$$
\Gamma_{\nu \nu}(t)=-\sum_{\kappa \neq \nu} \Gamma_{\kappa \nu}(t)
$$

This condition implies that one eigenvalue equals zero, i.e. $\Lambda_{1}=0$. Therefore,

$$
\rho_{\mu \mu}(t)=\rho_{\mu \mu}^{\infty}+\sum_{\nu=2}^{N} \sum_{\kappa=1}^{N} S_{\mu \nu}\left(S^{-1}\right)_{\mu \kappa} e^{\Lambda_{\nu}\left(t-t_{0}\right)} \rho_{\kappa \kappa}\left(t_{0}\right),
$$

with $\rho_{\mu \mu}^{\infty}=\sum_{\kappa=1}^{N} S_{\mu, 1}\left(S^{-1}\right)_{1, \kappa} \rho_{\kappa \kappa}\left(t_{0}\right)$ being the asymptotic population of the DVRstate $\left|q_{\mu}\right\rangle$. The rate which determines the dynamics over the largest time-scale is the quantum relaxation rate

$$
\Gamma \equiv \min \left\{\left|\Re\left(\Lambda_{\nu}\right)\right| ; \nu=2, \ldots, N\right\}
$$

where $\Lambda_{\nu}$ are the eigenvalues of the rate matrix and $\left|\Re\left(\Lambda_{\nu}\right)\right|$ are the non-zero absolute values of the real part of $\Lambda_{\nu}$.

In the next section we focus our study on the medium-short time behavior of the system, using the largest $\Lambda_{\nu}^{-1}$ as timescale to analyze the non-equilibrium dynamics of the quantum particle in the presence of thermal fluctuations.

\section{Results}

In this section, we study the time evolution of our quantum particle taking into account the 8 energy levels shown in Fig. 1. We restrict the study to the 8 lowest levels of the system, because we are interested in the dynamics of a particle that can not reach energy levels higher than the relative maximum of the potential. In particular, we intend to analyze the time behaviour of the populations for different values of the coupling strength, focusing on the time behaviour of the state $\left|q_{0}\right\rangle$ (left side well of the potential).

By using the DVR-state $\left|q_{\mu}\right\rangle$, as initial condition for the particle we choose the non-equilibrium position $q_{\text {start }}=0$. The corresponding state is given by

$$
\left|q_{\text {start }}\right\rangle=c_{1}\left|q_{1}\right\rangle+c_{2}\left|q_{2}\right\rangle
$$

with $c_{1}=0.745$ and $c_{2}=0.667$.

By integrating Eq. (10) for different values of the parameter $\eta$, which represents the intensity of the environmental noise, for each eigenstate $\left|q_{\mu}\right\rangle$ we obtain the time behaviour of the corresponding population $\rho_{q_{\mu}} \equiv \rho_{\mu \mu}$ (see Fig. 2). Moreover, by a simple change of basis, we calculate the time evolution of the populations also in the energy representation (see Fig. 3). As one can see from Eqs. (11), (13), for each value 

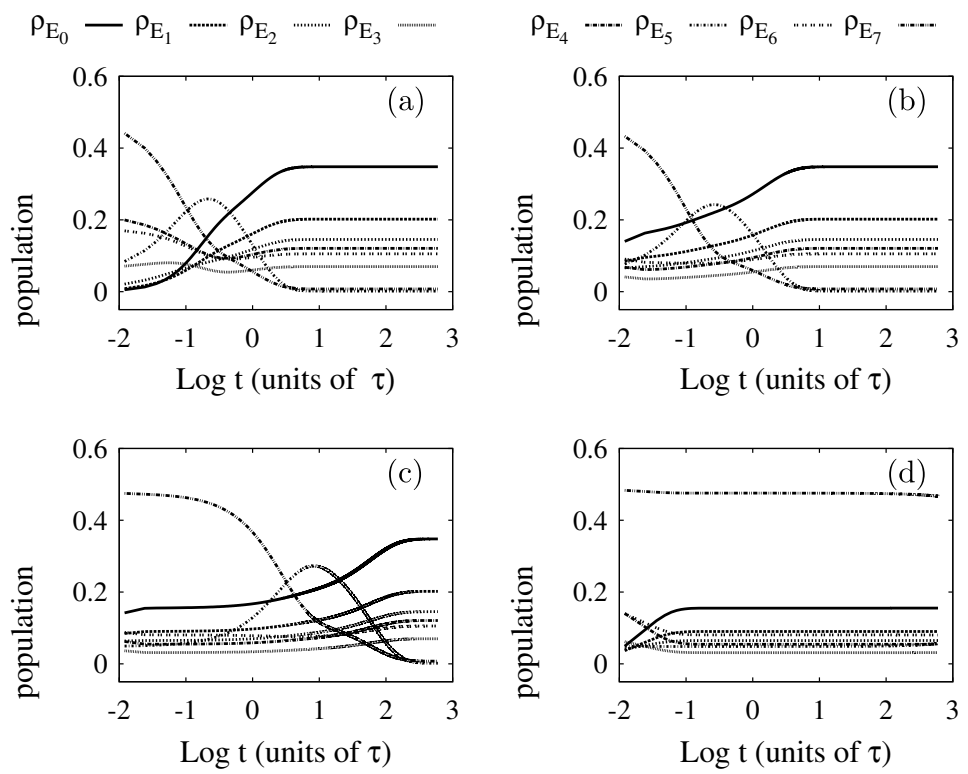

Fig. 3. Time evolution of the diagonal elements, $\rho_{E_{\mu}}(\mu=0,1, \ldots, 7)$, of the density matrix in energy representation. The matrix elements $\rho_{E_{\mu}}$ are the population distributions in the eight energy eigenstates considered. The time evolution is obtained for different values of the coupling strength: (a) $\eta=0.01$, (b) $\eta=0.4$, (c) $\eta=1$ and (d) $\eta=2.8$.

of $\eta$ there are $N$ relaxation times $\Lambda_{\mu}^{-1}$. Here, we consider the maximum of these relaxation times, and note that this time increases rapidly for larger values of $\eta$. Therefore, to describe the time evolution of the system for different values of $\eta$, we choose as time scale $\tau$ the largest of the relaxation times obtained for $\eta=0.01$ and calculate the evolution of the system for a maximum time $t=600 \tau$. This choice allows to follow the transient dynamics of the system for low and intermediate values of the coupling constant (see panels a, b, c in Figs. 2 and 3). For higher values of $\eta$ the system can not reach the regime condition, because of the presence of relaxation times longer than the maximum time chosen to calculate the numerical solution (see panel d in Figs. 2 and 3). This delay in the system dynamics can be explained by the quantum Zeno effect, responsible for the suppression of the tunnel effect. Moreover, we observe in Fig. 2 a nonmonotonic behaviour of the population $\rho_{q_{0}}$ as a function of the time. Finally, as a consequence of the quantum Zeno effect, the eigenstate $\left|q_{0}\right\rangle$ can be maximally populated at different times, varying the coupling strength and, therefore, the value of $\eta$. This could be useful in view of placing a quantum particle in a given position at a fixed time.

We note that it would be interesting to compare our results with those obtained in the case of a harmonic oscillator coupled with a thermal bath without any cutoff, as studied in previous works. ${ }^{21-23}$ On physical grounds we expect that the time behaviour of the purity of the system state is strictly connected with the relaxation rates. 
In our analysis the relaxation rates have been used to determine the timescale for obtaining the time evolution of the population distributions. Moreover, we found a freezing phenomenon of the state of the system due to the Zeno effect. ${ }^{24}$ Finally, we note that the complete description of the dynamics of our initial pure state should be obtained by following the time evolution of all elements of the density matrix as expressed by Eq. (8). This will be subject of future investigations.

\section{Conclusion}

In this work, we analyze the dynamics of a quantum particle subject to an asymmetric bistable potential and interacting with noisy environment. The study is performed exploiting the approach of the Feynman-Vernon functional ${ }^{18}$ within the framework of the discrete variable representation. ${ }^{19,20}$ By using the Caldeira-Leggett model,${ }^{4}$ we describe the transient dynamics of the system for different values of the coupling strength between the particle and the noisy environment, modelled as a thermal bath. Due to the quantum Zeno effect, responsible for the suppression of the tunnel effect, a delayed dynamics of the system is observed for higher values of the coupling strength. We find also that the metastable state inside the left side well of the potential can be populated at different times varying the value of the coupling strength.

\section{References}

1. Y. Nakamura, Yu. A. Pashkin and J. S. Tsai et al., Nature 398 (1999) 786.

2. D. Rakoczy, R. Heer, G. Strasser and J. Smoliner, Physica E 16 (2003) 129.

3. M. Thorwart, M. Grifoni and P. Hänggi, Ann. Phys. 293 (2001) 15.

4. A. O. Caldeira and A. L. Leggett, Phys. Rev. Lett. 46 (1981) 211.

5. A. J. Leggett, S. Chakravarty, A. T. Dorsey, M. Fisher, A. Garg and W. Zwerger, Rev. Mod. Phys. 59 (1987) 1.

6. M. Grifoni and P. Hänggi, Phys. Rep. 304 (1998) 229.

7. U. Weiss, Quantum Dissipative Systems (World Scientific, Singapore, 1999).

8. A. J. Leggett, Phys. Rev. B 30 (1984) 1208.

9. C.-H. Chou, T. Yu and B. L. Hu, Phys. Rev. E 77 (2008) 011112.

10. M. Rosenau da Costa, A. O. Caldeira, S. M. Dutra and H. Westfahl, Jr., Phys. Rev. A 61 (2000) 022107.

11. N. V. Prokofev and P. C. E. Stamp, Rep. Prog. Phys. 63 (2000) 669726.

12. H. P. Breuer and F. Petruccione, The Theory of Open Quantum Systems (Claredon Press, Oxford, 2006).

13. J. R. Friedman, V. Patel, W. Chen, S. K. Tolpygo and J. E. Lukens et al., Nature 406 (2000) 43.

14. J. M. Martinis, J. M. Martinis, S. Nam, J. Aumentado and C. Urbina et al., Phys. Rev. Lett. 89 (2002) 117901.

15. F. Grossmann, T. Dittrich, P. Jung and P. Hänggi, Phys. Rev. Lett. 67 (1991) 516.

16. R. Löfstedt and S. N. Coppersmith, Phys. Rev. Lett. 72 (1994) 1947.

17. L. Viola, E. Knill and S. Lloyd, Phys. Rev. Lett. 82 (1999) 2417.

18. R. P. Feynman and F. L. Vernon, Jr., Ann. Phys. 24 (1963) 118. 
19. D. O. Harris, G. G. Engerholm and W. D. Gwinn, J. Chem. Phys. 43 (1965) 1515.

20. M. Thorwart, M. Grifoni and P. Hänggi, Ann. Phys. 293 (2001) 15.

21. M. G. A. Paris, F. Illuminati, A. Serafini and S. De Siena, Phys. Rev. A 68 (2003) 012314.

22. A. Serafini, F. Illuminati, M. G. A. Paris and S. De Siena, Phys. Rev. A 69 (2004) 022318.

23. A. Serafini, M. G. A. Paris, F. Illuminati and S. De Siena, J. Opt. B 7 (2005) R19.

24. P. Facchi, S. Tasaki, S. Pascazio, H. Nakazato, A. Tokuse and D. A. Lidar, Phys. Rev. A 71 (2005) 022302. 